\title{
Cosmetotextiles - Sometimes the Simple Things Work
}

\section{Gadi Borkow}

Cupron Inc. Hameyasdim 44, Gibton 76910, Israel

*Corresponding author: Gadi Borkow, Cupron Inc. Hameyasdim 44, Gibton 76910, Israel, Tel: 972-546-611287; E-mail: gadi@cupron.com

Received date: January 01, 2016; Accepted date: January 02, 2016; Published date: January 04, 2016

Copyright: ( 2016 Borkow G. et al. This is an open-access article distributed under the terms of the Creative Commons Attribution License, which permits unrestricted use, distribution, and reproduction in any medium, provided the original author and source are credited.

\section{Editorial}

We all hope for healthy, smooth and beautiful looking skin. Many of us spend considerable amounts of money and time looking for products that can keep our skin looking young and radiant. The cosmetic industry is flourishing and new moisturizers, cleansers and beauty products constantly emerge in the market. The vast majority of products require active implementation - a daily care routine of cleansing and moisturizing of the facial skin, for example.

In recent years a new approach to skin care is emerging cosmetotextiles. This approach transforms daily ordinary textile products into cosmetically active products, eliminating the need to actively apply the cosmetic substance. We all use textiles, both during the day - our clothing, and during the night, our bed linens. These textiles are designed mainly to give us protection, warmth, and support. Novel technologies established in the last two decades gave rise to the development of sophisticated technical textiles, such as breathable textiles, sensing textiles, medical textiles, antimicrobial textiles, and more recently, cosmetotextiles.

Cosmetotextiles combine textile materials with cosmetic active substances. The cosmetic substance is affixed to the fabric of clothing or bedding so that when in contact with human body and skin the active substance is transferred from the textile onto the skin for cosmetic purposes [1]. Current cosmetotextiles in the market claim to be moisturizing, cellulite reducing, perfumed, body slimming, energizing, rejuvenating, refreshing, improving the firmness and elasticity of skin or reducing the appearance of fine lines and wrinkles.

The incorporation of cosmetic active ingredients into textiles can be achieved by several evolving technologies at the dope preparation before the fiber extrusion, at the yarn/fiber, fabric or end product stage [1]. One example is the incorporation of copper oxide micro-particles at the dope stage allowing the production of an array of textile products in which the copper oxide micro-particles are permanently embedded throughout the fabric fibers [2,3]. As copper plays an important role in skin formation, elasticity and firmness [4], this technology enabled the production of cosmetotextiles proven to reduce fine lines and wrinkles and to improve skin elasticity in double blind placebo controlled trials [5-7]. A second example is the incorporation of micro-capsules containing an active substance into textile products $[8,9]$. A determined amount of the active cosmetic substance, such as a moisturizer or vitamin C [10], is encapsulated and the microcapsule is attached to the textile fibers. Microencapsulation can be achieved by an array of methods, and the release of the active ingredient from the microcapsules occurs following heat, biodegradation, friction or pressure between the body and fabric during use, breaking the capsules into fragments and liberating the encapsulated active ingredients. A third example is coating of fabrics with cyclodextrin as a carrier for ingredient deliveries to the skin [11].
Each technique has its pros and cons. For example, the advantage of microencapsulation is the protection of the active substance from the environment, i.e., from heat, low or high $\mathrm{pH}$, oxidation, evaporation, and degradation. However, a potential disadvantage of microencapsulation is the potential allergenicity or toxicity of the microcapsules' shells once they are broken and released onto the skin.

Several questions need to be addressed when examining each cosmetotextile per se: is the claimed cosmetic effect achieved following the product use; have the claimed effects been demonstrated in clinical/cosmetic independent controlled studies; do you need to use the product daily to see an effect; for how long do you need to use the product to see an effect; is the cosmetic effect sustained following laundry (since cosmetotextiles are designed to be used as regular textiles, and as such, they are regularly laundered); for how many washings is the cosmetic effect maintained; is the product safe when used for prolonged periods of time; is it environmentally friendly, and what is the durability/shelf life of the product. In light of these questions, the regulatory bodies are developing appropriate standards to evaluate the efficacy, safety and durability of cosmetotextiles [1].

The number of companies producing cosmetotextiles and the number of different active ingredients being incorporated into textiles are constantly growing. The technologies used are being optimized to ensure sustainability and optimum cosmetic effects. Cosmetotextiles is a very attractive relatively new field gaining public acceptance by which regular textile products are converted into extraordinary cosmetic products. The idea of using your pillowcase or blouse and by doing so improving the well-being of your skin, not long ago was farfetched, but today it is a reality. Sometimes the simple things work.

\section{References}

1. Singh MK, Varun VK, Behera BK (2011) Cosmetotextiles: state of art. Fibres \& Textiles in Eastern Europe 19: 27-33.

2. Borkow G, Gabbay J (2004) Putting copper into action: copperimpregnated products with potent biocidal activities. FASEB J 18: 1728-1730.

3. Gabbay J, Mishal J, Magen E, Zatcoff RC, Borkow G, et al. (2006) Copper oxide impregnated textiles with potent biocidal activities. J Industrial Textiles 35: 323-335.

4. Borkow G (2014) Using copper to Improve the well-being of the skin. Curr Chem Biol 8: 89-102.

5. Baek JH, Yoo MA, Koh JS, Borkow G (2012) Reduction of facial wrinkles depth by sleeping on copper oxide-containing pillowcases: a double blind, placebo controlled, parallel, randomized clinical study. J Cosmet Dermatol 11: 193-200.

6. Borkow G, Gabbay J, Lyakhovitsky A, Huszar M (2009) Improvement of facial skin characteristics using copper oxide containing pillowcases: a double-blind, placebo-controlled, parallel, randomized study. Int J Cosmet Sci 31: 437-443. 
Citation: Borkow G (2016) Cosmetotextiles - Sometimes the Simple Things Work. J Cosmo Trichol 2: 1000e103. doi: 10.4172/2471-9323.1000e103

Page 2 of 2

7. Dykes P (2014) Increase in skin surface elasticity in normal voluntee subjects following the use of copper oxide impregnated socks. Skin Res Technol 21: 272-277.

8. Cheng SY, Yuen CWM, Kan CW, Cheuk KKL (2008) Development of cosmetic textiles using microencapsulation technology. RTJA 12: 41-51.

9. Casanova F, Santosa L (2015) Encapsulation of cosmetic active ingredients for topical application - a review. J Microencapsul 26: 1-17.
10. Cheng SY, Yuen MC, Kan CW, Cheuk KK, Chui CH, et al. (2009) Cosmetic textiles with biological benefits: gelatin microcapsules containing vitamin C. Int J Mol Med 24: 411-419.

11. Issazadeh-Baltorki H, Khoddami A (2014) Cyclodextrin-coated denim fabrics as novel carriers for ingredient deliveries to the skin. Carbohydr Polym 110: 513-517. 